Open Access

\title{
Naturally occurring mutations associated with resistance to HCV NS5B polymerase and NS3 protease inhibitors in treatment- naïve patients with chronic hepatitis $C$
}

\author{
Angela Costantino ${ }^{1 *}$ D , Enea Spada ${ }^{1}$, Michele Equestre ${ }^{2}$, Roberto Bruni ${ }^{1}$, Elena Tritarelli ${ }^{1}$, Nicola Coppola ${ }^{3}$,
} Caterina Sagnelli, Evangelista Sagnelli ${ }^{3}$ and Anna Rita Ciccaglione ${ }^{1}$

\begin{abstract}
Background: The detection of baseline resistance mutations to new direct-acting antivirals (DAAs) in HCV chronically infected treatment-naïve patients could be important for their management and outcome prevision. In this study, we investigated the presence of mutations, which have been previously reported to be associated with resistance to DAAs in HCV polymerase (NS5B) and HCV protease (NS3) regions, in sera of treatment-naïve patients.

Findings: HCV RNA from 152 naive patients ( $84 \%$ Italian and $16 \%$ immigrants from various countries) infected with different HCV genotypes $(21,1 \mathrm{a} ; 21,1 \mathrm{~b} ; 2,2 \mathrm{a} ; 60,2 \mathrm{c} ; 22,3 \mathrm{a} ; 25,4 \mathrm{~d}$ and $1,4 \mathrm{k})$ was evaluated for sequence analysis. Amplification and sequencing of fragments in the NS5B (nt 8256-8640) and NS3 (nt 3420-3960) regions of HCV genome were carried out for 152 and 28 patients, respectively. The polymorphism C316N/H in NS5B region, associated with resistance to sofosbuvir, was detected in 9 of the 21 (43\%) analysed sequences from genotype 1b-infected patients. Naturally occurring mutations V36L, and M175L in the NS3 protease region were observed in $100 \%$ of patients infected with subtype 2c and 4.

Conclusion: A relevant proportion of treatment naive genotype $1 \mathrm{~b}$ infected patients evaluated in this study harboured N316 polymorphism and might poorly respond to sofosbuvir treatment. As sofosbuvir has been approved for treatment of HCV chronic infection in USA and Europe including Italy, pre-treatment testing for N316 polymorphism on genotype $1 \mathrm{~b}$ naïve patients should be considered for this drug.
\end{abstract}

Keywords: Hepatitis C virus, Mutations, Antiviral treatment, Genotype, Italy

\section{Background}

Hepatitis $\mathrm{C}$ virus (HCV) is a global health problem. Approximately $3 \%$ of the world population is infected with $\mathrm{HCV}$, the leading cause of liver cirrhosis, hepatic decompensation, development of hepatocellular carcinoma (HCC) and liver transplantations worldwide [1]. HCV is characterized by a high genetic heterogeneity and is classified in 7 genotypes and numerous subtypes [2]. Some subtypes are ubiquitous while others circulate in particular regions [3]. In Italy, the prevalence of $\mathrm{HCV}$ is

\footnotetext{
* Correspondence: angela.costantino@iss.it

'Department of Infectious, Parasitic and Immune-Mediated Diseases, Viral Hepatitis Unit, Istituto Superiore di Sanità, Viale Regina Elena, 299-00161 Rome, Italy

Full list of author information is available at the end of the article
}

declining. Recent studies carried out among general population in both northern and southern Italy have reported an anti-HCV prevalence ranging from 2.6 to 5.7 [4-6]. However it is a common opinion among researchers that at present in Italy the prevalence of $\mathrm{HCV}$ chronic carriers is about $1-1.5 \%$. The most frequent genotypes circulating in general population are $1 \mathrm{~b}, 2 \mathrm{c}$ and, to a less extent, $3 \mathrm{a}$, while the prevalence of genotype 4 has increased in recent years, in southern regions [6-8]. Pegylated-Interferon plus ribavirin (peg-IFN-RBV) has been the standard-of-care treatment for chronic hepatitis $\mathrm{C}$ for many years, although it had some disadvantages, such as a suboptimal therapy response rate in some patients' subsets (genotype 1 and 4 infections, advanced 
liver fibrosis) and side effects often resulting in poor tolerability and treatment contraindication [9]. Recently, new anti-HCV drugs, termed direct-acting antiviral agents (DAAs) have been developed. DAAs target different regions of $\mathrm{HCV}$ genome and can be distinguished in NS3/4A protease inhibitors (PIs), NS5A inhibitors, NS5B nucleoside (NPIs) and non-nucleoside polymerase inhibitors (NNPIs). Boceprevir and Telaprevir, first generation NS3 PIs, were approved in the 2011 in United States by Food and Drug Administration and Europe (FDA) by European Medicines Agency (EMA) for therapy of chronic hepatitis C. These drugs, however, administered in combination with peg-IFN and ribavirin were hampered by a low efficacy against non-1 genotypes, serious adverse reactions and low genetic barrier to resistance. In addition to the first generation PIs, two new NS3 PIs (Simeprevir and Faldaprevir) one NS5A inhibitor (Dataclasvir) and one NS5B NPI (Sofosbuvir) have been recently (FDA, EMA, 2014) approved for treatment of chronic hepatitis C [10]. The most recent DAAs increase response rates, allow shortened and simplified regimens and have a higher genetic barrier to resistance [11]. However, the effectiveness of new DAAs may be affected by the development of drug-resistance mutations. Due to the high sequence diversity, high replication rate and low fidelity of HCV RNA-polymerase, numerous variants, defined as quasispecies, exist in vivo in a $\mathrm{HCV}$ infected patient [12]. Some of these variants can carry amino-acid substitutions which determine conformation changes of a drug binding site, thus causing resistance during therapy $[13,14]$. These drug resistance substitutions, which usually emerge after a few days of DAAs treatment and are responsible for treatment failure (particularly with first generation drugs) $[15,16]$ or hyporesponsiveness to treatment, can also be found in $\mathrm{HCV}$ infected treatment-naïve patients [17-23]. These naturally resistant variants have been reported to occur at variable frequencies and are genotype/subtype dependent. In fact, the frequency of natural resistance mutations to first generation NS3 PIs is lower in genotype $1 \mathrm{~b}$ compared to genotype 1a patients [19]. Resistance mutations to NS3 PIs in treatment-naïve patients infected with non-1 genotypes have been investigated in several studies, but only one of them detected two principal mutations (V158M for genotype 2c and D168E for genotype 4) in a significant number of patients infected with genotypes $2 \mathrm{c}$ and 4 [23]. On the contrary, many substitutions associated with resistance to NS3 PIs have been reported for genotype 1 [24]. The S282T mutation in NS5B polymerase region, identified in vitro [25] and in vivo in a $2 \mathrm{~b}$ infected patient who failed therapy during a clinical trial $[26,27]$, is the only mutation so far surely associated with resistance to sofosbuvir. Indeed, several other NS5B substitutions have also been suggested as responsible for sofosbuvir treatment failure [28]. In particular, a baseline NS5B polymorphism at position 316 has been potentially associated with reduced response rates to sofosbuvir in genotype $1 \mathrm{~b}$ patients [29]. The aim of this study was to investigate the presence of variants resistant to DAAs in the NS5B polymerase and NS3 serine protease regions by analysing patients with chronic hepatitis $\mathrm{C}$ who had not been treated with any DAAs.

\section{Materials and methods}

This study included 152 DAA-naïve patients chronically infected with HCV genotype 1a $(n=21), 1 \mathrm{~b}(n=21), 2 \mathrm{a}$ $(n=2), 2 \mathrm{c}(n=60), 3 \mathrm{a}(n=22), 4 \mathrm{~d}(n=25)$ and $4 \mathrm{k}(n=$ 1) $[6-8]$, and analysed for NS5B region (nt 8256-8640) using primers and amplification conditions reported elsewhere [4, 6-8]. For 28 (11, 1b; 11, 2c and 6, 4d) of these 152 patients, HCV-NS3 region (3420-3960) was also amplified by nested PCR using in the first round primers and amplification conditions previously reported [30]. In the second round, new designed primers and a different thermal cycle program were used, while all other amplification conditions (buffer, primers' concentration, etc.) were the same as those used in the first

Table 1 Primers used for HCV NS3 protease gene amplification

\begin{tabular}{|c|c|c|c|c|}
\hline $\begin{array}{l}\text { HCV } \\
\text { genotype }\end{array}$ & I-PCR primers & II-PCR primers & $5^{\prime}-3^{\prime}$ sequence ${ }^{b}$ & H77 location \\
\hline $1 \mathrm{~b}$ & ${\text { MarsF2 }{ }^{\mathrm{a}} \text { MarsR1 }}^{\mathrm{a}}$ & NS31b F ${ }^{b}$ MarsR2 $^{a}$ & 5'- TRCCHGTCTCCGCCCGVAG-3'C & $3291-33104215-42373340-33584035-4054$ \\
\hline $2 c$ & ${\text { MarsF1 } 1^{\mathrm{M}} \text { MarsR1 }}^{\mathrm{a}}$ & NS3 2c F NS3 2c R & $\begin{array}{l}\text { 5'- TGGGCCCTGCTGATGGATAC-3' } 5^{\prime}- \\
\text { GTAGGTCTGGGGCACAGCTG-3' }\end{array}$ & $3291-33104215-42373376-33954010-3991$ \\
\hline $4 d$ & $\mathrm{G} 4 \mathrm{~F} 1^{\mathrm{a}} \mathrm{G} 4 \mathrm{R} 1^{\mathrm{a}}$ & NS3 4d F ${ }^{\mathrm{b}} \mathrm{NS3} 4 \mathrm{~d} \mathrm{R}^{\mathrm{b}}$ & $\begin{array}{l}\text { 5'-ATGCGCACRCYAYGAAGGG-3'e } 5^{\prime}- \\
\text { GGCACGGCAGGAGGAGTGGA-3' }\end{array}$ & $3369-33883997-40183388-34074000-3981$ \\
\hline
\end{tabular}

\footnotetext{
${ }^{a}$ Previously published primers (Vallet S [30])

${ }^{b}$ Primers selected by alignment of sequences from Los Alamos databank

'Amplification conditions in the second PCR for genotype 1b, were: $2^{\prime}$ at $94{ }^{\circ} \mathrm{C}$, and then 30 cycles at $94^{\circ} \mathrm{C}$ for $30^{\prime \prime}, 60^{\circ} \mathrm{C}$ for $30^{\prime \prime}$ and $72{ }^{\circ} \mathrm{C}$ for $45^{\prime \prime}$, plus extension at $72{ }^{\circ} \mathrm{C}$ for $7^{\prime}$

${ }^{d}$ Amplification conditions in the second PCR for genotype $2 \mathrm{C}$, were: $2^{\prime}$ at $94^{\circ} \mathrm{C}$, and then 30 cycles at $94{ }^{\circ} \mathrm{C}$ for $30^{\prime \prime}, 62^{\circ} \mathrm{C}$ for $30^{\prime \prime}$ and $72{ }^{\circ} \mathrm{C}$ for $45^{\prime \prime}$, plus extension at $72{ }^{\circ} \mathrm{C}$ for $7^{\prime}$

${ }^{e}$ Amplification conditions in the second PCR for genotype 4, were: $2^{\prime}$ at $94^{\circ} \mathrm{C}$, and then 30 cycles at $94{ }^{\circ} \mathrm{C}$ for $30^{\prime \prime}, 60^{\circ} \mathrm{C}$ for $40^{\prime \prime}$ and $72^{\circ} \mathrm{C}$ for $45^{\prime \prime}$, plus extension at $72{ }^{\circ} \mathrm{C}$ for $7^{\prime}$
} 
Table 2 Aminoacid substitutions in HCV NS5B polymerase region associated with resistance to DAAs ${ }^{\mathrm{a}}$ in treatment-naive patients

\begin{tabular}{|c|c|c|c|c|c|c|c|c|c|c|c|}
\hline$\overline{R^{b}}$ & $P^{c}$ & $D^{d}$ & $M^{e}$ & Drug & $\mathrm{G} 1 \mathrm{a}(n=21)$ & $\mathrm{G} 1 \mathrm{~b}(n=21)$ & G $2 \mathrm{a}(n=2)$ & $\mathrm{G} 2 \mathrm{c}(n=60)$ & G 3a $(n=20)$ & $\mathrm{G} 4 \mathrm{~d}(n=25)$ & $\mathrm{G} 4 \mathrm{k}(n=1)$ \\
\hline NS5B & 316 & $C$ & $\mathrm{~N}, \mathrm{Y}, \mathrm{H}$ & $\begin{array}{l}\text { Sofosbuvir, } \\
\text { Mericitabine }\end{array}$ & . & ${ }^{*} \mathrm{C}(57) / \mathrm{N}(38), \mathrm{H}(5)$ & . & . & . & . & . \\
\hline NS5B & 282 & S & $\mathrm{T}$ & $\begin{array}{l}\text { Sofosbuvir, } \\
\text { Mericitabine }\end{array}$ & • & - & $\cdot$ & - & - & - & $\cdot$ \\
\hline NS5B & 320 & I & & $\begin{array}{l}\text { Sofosbuvir, } \\
\text { Mericitabine }\end{array}$ & • & • & $\cdot$ & • & • & $\cdot$ & • \\
\hline NS5B & 321 & V & & $\begin{array}{l}\text { Sofosbuvir, } \\
\text { Mericitabine }\end{array}$ & - & - & • & • & - & - & - \\
\hline
\end{tabular}

a direct acting antiviral agents

$\mathrm{R}^{\mathrm{b}}$, Region; $\mathrm{P}^{\mathrm{c}}$, Position aa; $\mathrm{D}^{\mathrm{d}}$, Dominant aa; $\mathrm{M}^{\mathrm{e}}$, Mutant aa; (Donaldson, [29]; Lam, [25]; Tong, [28])

*Percentage of sequences with substitutions

-The dominant residue is found in $100 \%$ of analyzed sequences for each genotype

PCR round (Table 1). An automated DNA sequencer (Beckman Coulter, Inc., Fullerton, CA) was used for sequencing. Nucleotide sequences were assembled using Chromas software, and aligned using ClustalW package.

\section{Results}

A total of 152 patients (81 males and 55 females; median age 47, range 20-90 years) were analysed. About $84 \%(127 / 152)$ of these patients were of Italian origin, while the remaining 25 patients were immigrants from various countries. From sera of these patients were obtained 152 NS5B and 28 NS3 sequences. All aminoacid substitutions in NS5B polymerase and NS3 protease regions potentially associated with resistance to DAAs in $\mathrm{HCV}$ - infected treatment-naive patients in this study, are shown in Tables 2 and 3, respectively. The NS5B polymerase fragment of all analysed genotypes showed no mutation known to induce high level of resistance to sofosbuvir in vitro (S282T), as well as other mutations recently regarded as responsible for sofosbuvir treatment failure in clinical trials (V321I/A, L320F/C) [22]. Whereas, the polymorphism $\mathrm{C} 316 \mathrm{~N} / \mathrm{H}$, potentially associated with reduced response rates to sofosbuvir in genotype $1 \mathrm{~b} \mathrm{HCV}$ chronically infected patients [23], was found in 9 of
21 (43\%) analysed $1 \mathrm{~b}$ sequences (C316N and $\mathrm{C} 316 \mathrm{H}$ polymorphisms were detected in 8 and in 1 patients, respectively). No substitutions conferring resistance to both first generation and new NS3 PIs (Simeprevir and Faldaprevir), were observed in the NS3 region of genotype $1 \mathrm{~b}$ sequences. Instead, the V36L and M175L substitutions, know to induce decreased susceptibility exclusively to first generation PIs in genotype 1 infections, were naturally present in NS3 region of genotype $2 \mathrm{c}$ and $4 \mathrm{~d}$ sequences.

Finally, several polymorphisms not associated with resistance to DAAs and already reported by others [18] were observed in NS3 region of our analysed-genotypes (Tables 4, 5 and 6).

\section{Discussion}

This study reports the results of the HCV sequencing from 152 treatment-naïve patients chronically infected with different $\mathrm{HCV}$ genotypes. In these patients, we evaluated the presence of known drug resistance mutations focusing on NS5B polymerase and NS3 protease regions. Aminoacid substitutions, such as V36L and M175L, associated with HCV-genotype 1 PIs resistance in vivo and in vitro, were naturally observed in $100 \%$ of our patients

Table 3 Aminoacid substitutions in HCV NS3 protease region associated with resistance to DAAs ${ }^{a}$ in treatment-naive patients

\begin{tabular}{|c|c|c|c|c|c|c|c|}
\hline$\overline{R^{b}}$ & $P^{c}$ & $D^{d}$ & $M^{e}$ & Drug & $\mathrm{G} 1 \mathrm{~b}(n=11)$ & $\mathrm{G} 2 \mathrm{c}(n=11)$ & $\mathrm{G} 4 \mathrm{~d}(n=6)$ \\
\hline NS3 & 16 & C & S & ACH806 & ${ }^{*} C / R(9)$ & ${ }^{*} A(100)$ & ${ }^{*} T(100)$ \\
\hline NS3 & 36 & V & $\mathrm{A}, \mathrm{M}, \mathrm{G}, \mathrm{L}$ & Telaprevir, Boceprevir & $* V(100)$ & $L(100)^{f}$ & $L(100)^{f}$ \\
\hline NS3 & 54 & $\mathrm{~T}$ & $A, S$ & Telaprevir, Boceprevir & $\cdot$ & $\cdot$ & $\cdot$ \\
\hline NS3 & 155 & $\mathrm{R}$ & $\mathrm{K}, \mathrm{T}, \mathrm{I}, \mathrm{MG}, \mathrm{L}, \mathrm{S}, \mathrm{Q}$ & Telaprevir, Boceprevir & · & $\cdot$ & · \\
\hline NS3 & 156 & A & $\mathrm{S}, T, \mathrm{~V}, \mathrm{I}$ & Telaprevir, Boceprevir & $\cdot$ & $\cdot$ & $\cdot$ \\
\hline NS3 & 168 & $\mathrm{D}$ & $\mathrm{Q}, \mathrm{A}, \mathrm{V}, \mathrm{E}, \mathrm{T}$ & TMC435,R7227,MK7009, BI201335, BILN2061 & $\cdot$ & $\cdot$ & $\cdot$ \\
\hline NS3 & 175 & M & L & Telaprevir & ${ }^{*} \mathrm{M}(100)$ & $L(100)^{f}$ & $L(100)^{f}$ \\
\hline
\end{tabular}

a direct acting antiviral agents

$\mathrm{R}^{\mathrm{b}}$, Region; $\mathrm{P}^{\mathrm{c}}$,Position aa; $\mathrm{D}^{\mathrm{d}}$,Dominant aa; $\mathrm{M}^{\mathrm{e}}$, Mutant aa; (Lopez-Labrador, 2008; Lentz, 2010; Kieffer [15], Halfon [13], Kuntzen [19])

*Percentage of sequences with substitutions

-The dominant residue is found in $100 \%$ of analyzed sequences for each genotype

${ }^{f}$ Substitution found is associated with resistance in genotype $1 \mathrm{~b}$ 
Table 4 Polymorphic sites in the HCV 1b NS3 protease region found in this study

\begin{tabular}{llll}
\hline a Position & bSubtype 1b & aPosition & bSubtype 1b \\
\hline 7 & S7/A (6) & 114 & V114/I (11) \\
14 & L14/N (1) & 122 & S122/T (1) \\
30 & D30/E (10) & 128 & S128/Y (1) \\
48 & V48/I (6) & 131 & P131/T (1) \\
51 & V51/A (1) & 132 & I132N/L (5) \\
56 & Y56/F (4) & 142 & P142/L (1) \\
61 & S61/P/T (5) & 150 & V150/A (9) \\
83 & V83 /I (1) & 170 & I170/N (5) \\
86 & P86/Q (11) & 178 & T178/N (2) \\
87 & A87/S (2) & & \\
94 & M94/L (10) & & \\
107 & V107/I (1) & & \\
\hline
\end{tabular}

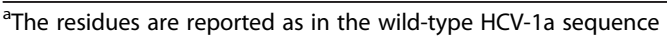

${ }^{\mathrm{b}}$ The number of patients with mutant HCV strains is indicated in brackets

infected with genotype $2 \mathrm{c}$ and $4 \mathrm{~d}$. The impact of this latter finding on therapy is unclear. Indeed, boceprevir and telaprevir showed less efficacy against genotype 2, and almost no efficacy against other non-1 genotypes, including genotype 4 . We cannot exclude that the reported low efficacy of boceprevir and telaprevir against genotype 2 and 4 is influenced by the presence of these natural polymorphisms detected by us and others [23]. The mutations in the NS3 region are rare to detect in naïve patients. Unfortunately, for tecnical reason the sequence analysis of the NS3 region in this study was limited to 28 serum samples only. However, these descriptive frequency data could be useful for further studies that analyse this HCV RNA region in the prediction of $\mathrm{HCV}$ antiviral therapy outcome. With regard to the presence of pre-treatment sofosbuvir resistance mutations, the S282T variant was not found in any of our patients, regardless of genotype. This is in agreement with literature data reporting that S282T variants are mainly found in vitro [25]. It is likely that our negative result, regarding the detection of this mutation, may be

Table 5 Polymorphic sites not associated with resistance to DAAs in the HCV 2c NS3 protease

\begin{tabular}{ll}
\hline Position NS3 $^{\mathrm{a}}$ & Subtype $2 \mathrm{C}^{\mathrm{b}}$ \\
\hline 15 & D15G/S (9) \\
33 & V33I (3) \\
35 & V35I (1) \\
134 & S134T (4) \\
146 & P146S (1) \\
177 & V177I (1) \\
\hline
\end{tabular}

${ }^{a}$ The residues are reported as in the wild-type HCV-1a sequence

${ }^{\mathrm{b}}$ The number of patients with mutant HCV strains is indicated in brackets
Table 6 Polymorphic sites in the HCV-4 NS3 protease not resistance-associated found in this study

\begin{tabular}{llll}
\hline PPositions & bSubtype 4 & aPositions & bSubtype 4 \\
\hline 12 & G12/R (1) & 98 & T98/A (1) \\
13 & L13/M (8) & 102 & A102/S (8) \\
14 & F14/L (8) & 105 & Y105/F (7) \\
15 & S15/G (7) & 114 & I114/ (8) \\
18 & V18/I (8) & 122 & T122/N (1) \\
24 & R24/K (1) & 127 & L127/I (5) \\
33 & V33/I (4) & 133 & S133/A (1) \\
47 & A47/S (3) & 134 & I134/T (8) \\
48 & V48/I (7) & 147 & M147/L (8) \\
61 & A61/S (8) & 150 & R150/A (8) \\
92 & R92/K (8) & 172 & V172/I (1) \\
95 & A95/T (1) & 179 & M179/A (1) \\
\hline
\end{tabular}

${ }^{a}$ The residues are reported as in the wild-type HCV-1a sequence

${ }^{b}$ The number of patients with mutant HCV strains is indicated in brackets

also due to the limits of the sequencing method used in this study. Indeed, the Sanger method can potentially misrepresent the totality of viral quasispecies harboured by an HCV infected patient [27]. In fact, in a recent study the S282T variant has been detected by deep sequencing at $0.05 \%$ of viral sequences at baseline in a genotype $2 \mathrm{~b}$ infected subject who relapsed following 12 weeks of SOF monotherapy [27]. Besides, other S282 variants (S282R/G/C) have been identified in naïve subjects [31].

Recently, novel (treatment-emergent) NS5B mutations (L159F, L320F and V321A) were detected in HCV infected patients who failed sofosbuvir treatment [29]. Importantly, a baseline polymorphism at position 316 has been associated with sofosbuvir treatment failure in genotype $1 \mathrm{~b}$ infected patients [29]. It has been reported that C316 mutation is positioned closed to the catalytic triad of the NS5B polymerase and changes at this position can prevent binding of sofosbuvir [29]. We have found substitution $316 \mathrm{~N} / \mathrm{H}$ in $43 \%$ of genotype $1 \mathrm{~b}$ sequences. The same position, as well as positions 320 and 321 , was highly conserved (100 \%) in the other analysed genotypes. The identification of baseline resistance mutations to DAAs could be important for patients' management and outcome prevision. In this regard, the detection of $316 \mathrm{~N} / \mathrm{H}$ polymorphism in $43 \%$ of our $1 \mathrm{~b}$ naive infected patients represents the most relevant finding of this study since these patients might poorly respond to sofosbuvir therapy. Indeed, a recent study reported that baseline polymorphisms at position 316 were potentially associated with reduced response rates in HCV genotype $1 \mathrm{~b}$ subjects [29]. In conclusion, pretreatment testing for $\mathrm{C} 316 \mathrm{~N}$ polymorphism on $\mathrm{HCV}$ genotype $1 \mathrm{~b}$ seems to be indicated for naïve patients considered for treatments including sofosbuvir. 


\begin{abstract}
Abbreviations
HCV: Hepatitis C virus; IFN: Interferon; RBV: Ribavirin; DAAs: Direct-acting antiviral agents; PIs: Protease inhibitors; NS5B-NPIs: NS5B nucleoside polymerase inhibitors; NS5B -NNPIs: NS5B non-nucleoside polymerase inhibitors; NS3: Non-structural region 3; NS5B: Non-structural region 5B.
\end{abstract}

\section{Competing interests}

All authors declare no conflict of interest.

\section{Authors' contributions}

AR C study concept and design, analysis and interpretation of data, and critical revision of the manuscript for important intellectual content; AC molecular analysis, interpretation of the data, writing the manuscript, and critical revision of the manuscript for important intellectual content; ES interpretation of data, contribution in writing the manuscript, and critical revision of the manuscript for important intellectual content; RB interpretation of data, and critical revision of the manuscript for important intellectual content; ME interpretation of data, and critical revision of the manuscript for important intellectual content. All authors read and approved the final manuscript for publication.

\section{Acknowledgements}

This study was funded by the Italian Ministry of Health (National Centre for Disease Prevention and Control, Fasc. 3 M58).

\section{Author details}

${ }^{1}$ Department of Infectious, Parasitic and Immune-Mediated Diseases, Viral Hepatitis Unit, Istituto Superiore di Sanità, Viale Regina Elena, 299-00161 Rome, Italy. ${ }^{2}$ Department of Cell Biology and Neurosciences, Istituto Superiore di Sanità, Rome, Italy. ${ }^{3}$ Department of Mental Health and Public Medicine, Section of Infectious diseases, Second University of Naples, Naples, Italy. ${ }^{4}$ Department of Experimental Medicine and Surgery F. Magrassi and A. Lanzara, Second University of Naples, Naples, Italy.

\section{Received: 5 June 2015 Accepted: 30 October 2015}

\section{Published online: 14 November 2015}

\section{References}

1. World Healt Org: Hepatitis C fact sheet $N^{\circ}$ 164. June 2011 available from http://www.who.int/mediacentre/factsheets/fs164/en/.

2. Simmonds $P$. The origin of hepatitis $C$ virus. Curr Top Microbiol Immunol. 2013:369:1-15.

3. Lavanchy D. Evolving epidemiology of hepatitis C virus. Clin Microbiol Infect. 2011:17:107-15.

4. Fabris P, Baldo V, Baldovin T, Bellotto E, Rassu M, Trivello R, et al. Changing epidemiology of HCV and HBV infections in Northern Italy: a survey in the general population. J Clin Gastroenterol. 2008;42:527-32.

5. Cozzolongo R, Osella AR, Elba S, Petruzzi J, Buongiorno G, Giannuzzi V, et al. Epidemiology of HCV infection in the general population: a survey in a southern Italian town. Am J Gastroenterol. 2009;104:2740-46.

6. Guadagnino V, Stroffolini T, Caroleo B, Menniti Ippolito F, Rapicetta M, Ciccaglione $A R$, et al. Hepatitis $C$ virus infection in an endemic area of Southern Italy 14 years later: evidence for a vanishing infection. Dig and Liver Dis. 2013;45:403-7.

7. Marascio N, Ciccozzi M, Equestre M, Lo Presti A, Costantino A, Cella E, et al Back to the origin of HCV 2c subtype and spreading to the Calabria region (Southern Italy) over the last two centuries: a phylogenetic study. Infect Genet Evol. 2014;26:352-8.

8. Ciccozzi M, Equestre M, Costantino A, Marascio N, Quirino A, Lo Presti A et al. Hepatitis C virus genotype $4 d$ in Southern Italy: reconstruction of its origin and spread by a phylodynamic analysis. J Med Virol. 2012;84:1613-9.

9. EASL (2011): 46th annual congress, March 30 - April 3, 2011 available from http://www.hepmag.com/archive/currentNews_2596.shtml.

10. Aghemo A, De Francesco R. New horizons in hepatitis $C$ antiviral therapy with direct-acting antivirals. Hepatol. 2013;58:428-38.

11. Gutierrez JA, Lawitz EJ, Poordad F. Interferon-free, direct-acting antiviral therapy for chronic hepatitis C. J Viral Hepat. 2015 Jun 17. doi:10.1111/jvh.12422

12. Ogata N, Alter HJ, Miller RH, Purcell RH. Nucleotide sequence and mutation rate of the $\mathrm{H}$ strain of hepatitis $\mathrm{C}$ virus. Proc Natl Acad Sci U S A. 1991;88:3392-6.
13. Halfon $P$, Locarnini $S$. Hepatitis $C$ virus resistance to protease inhibitors. J Hepatol. 2011;55:192-206.

14. Wyles DL. Antiviral resistance and the future landscape of hepatitis C virus infection therapy. J Infect Dis. 2013;207 Suppl 1:S33-9.

15. Kieffer TL, Sarrazin C, Miller JS, Welker MW, Forestier N, Reesink HW, et al. Telaprevir and pegylated interferon-alpha-2a inhibit wild-type and resistant genotype 1 hepatitis C virus replication in patients. Hepatol. 2007:46:631-39.

16. Sarrazin C, Zeuzen S. Resistance to direct antiviral agents in patients with hepatitis C virus infection. Gastroenterol. 2010;138:447-62.

17. Paolucci S, Fiorina L, Mariani B, Gulminetti R, Novati S, Barbarini G, et al Naturally occurring resistance mutations to inhibitors of HCV NS5A region and NS5B polymerase in DAA treatment-naive patients. Virol J. 2013;10:355

18. Bartels DJ, Sullivan JC, Zhang EZ, Tigges AM, Dorrian JL, De Meyer S, et al. Hepatitis $C$ virus variants with decreased sensitivity to direct-acting antivirals (DAAs) were rarely observed in DAA-Naïve patients prior to treatment. J Virol. 2013:87:1544-53.

19. Kuntzen T, Timm J, Berical A, Lennon N, Berlin AM, Young SK, et al. Naturally occurring dominant resistence mutations to hepatitis $\mathrm{C}$ virus protease and polymerase inhibitors in treatment-naïve patients. Hepatol. 2008;48:1769-78.

20. Applegate TL, Gaudieri S, Plauzolles A, Chopra A, Grebely J, Lucas M, et al. Naturally occurring dominant drug resistance mutations occur infrequently in the setting of recently acquired hepatitis C. Antivir Ther. 2015;20:199-208.

21. López-Labrador FX, Moya A, Gonzàlez-Candelas F. Mapping natural polymorphisms of hepatitis C virus NS3/4A protease and antiviral resistance to inhibitors in worldwide isolates. Antivir Ther. 2008;13:481-94.

22. Lenz $\mathrm{O}$, Verbinnen $\mathrm{T}$, Lin $\mathrm{Tl}$, Vijgen $\mathrm{L}$, Cummings MD, Lindberg J, et al. In vitro resistance profile of the hepatitis C virus NS3/4A protease inhibitor TMC435. Antimicrob Agents Chemother. 2010;54:1878-87.

23. Paolucci S, Fiorina L, Piralla A, Gulminetti R, Novati S, Barbarini G, et al. Naturally occurring mutations to HCV protease inhibitors in treatment-naive patients. Virol J. 2012;9:245

24. Wu S, Kanda T, Nakamoto S, Imazeki F, Yokosuka O. Hepatitis C virus protease inhibitor-resistance mutations: our experience and review. World J Gastroenterol. 2013;21:198940-8

25. Lam AM, Espiritu C, Bansal S, Micolochick Steuer HM, Niu C, Zennou V, et al. Genotype and subtype profiling of PSI-7977 as a nucleotide inhibitor of hepatitis C virus. Antimicrob Agents Chemother. 2012;56:3359-68.

26. Gane EJ, Stedman CA, Hyland RH, Ding X, Svarovskaia E, Symonds WT, et al. Nucleotide polymerase inhibitor sofosbuvir plus ribavirin for hepatitis C. N Engl J Med. 2013;368:34-44

27. Hedskog C, Dvory-Sobol H, Gontcharova V, Martin R, Ouyang W, Han B, et al. Evolution of the HCV viral population from a patient with S282T detected at relapse after sofosbuvir monotherapy. J Viral Hepat. 2015 Mar 18. doi: 10.1111/jvh.12405.

28. Tong X, Le Pogam S, Li L, Haines K, Piso K, Baronas V, et al. In vivo emergence of a novel mutant L159F/L320F in the NS5B polymerase confers low-level resistance to the HCV polymerase inhibitors mericitabine and sofosbuvir. J Infect Dis. 2014:209:668-75.

29. Donaldson EF, Harrington PR, O' Rear JJ, Naeger LK. Clinical evidence and bioinformatics characterization of potential hepatitis $C$ virus resistance pathways for sofosbuvir. Hepatol. 2015;61:56-65.

30. Vallet S, Viron F, Henquell C, Le Guillou-Guillemette H, Lagathu G, Abravanel $F$, et al. NS3 protease polymorphism and natural resistence to protease inhibitors in French patients infected with HCV genotype 1-5. Antiviral Therpy. 2011;16:1093-102.

31. Ji H, Kozak RA, Biondi MJ, Pilon R, Vallee D, Liang BB, et al. Next generation sequencing of the hepatitis C virus NS5B gene reveals potential novel S282 drug resistance mutations. Virol. 2015;477:1-9. 